\title{
Cor triatriatum sinister, not mitral stenosis, in an adult with previous Sydenham's chorea: diagnosis and preoperative assessment by cross sectional echocardiography
}

Mark A de Belder, Vincenzo Argano, Christopher J Burrell

\begin{abstract}
In cor triatriatum sinister, one of the rarest congenital cardiac anomalies, a membrane divides the left atrium into a pulmonary venous component above and the vestibule below. The importance of the anomaly lies in the effects of the resultant pulmonary venous obstruction that usually present in the first year of life and can mimic obstructed total anomalous venous drainage or congenital mitral stenosis. A case presented as mitral stenosis in the third decade of life, ten years after a well documented episode of Sydenham's chorea. The diagnosis was made rapidly by transthoracic echocardiography and transoesophageal echocardiography was used for complete assessment. Cardiac catheterisation added nothing to the non-invasive diagnosis or the preoperative assessment. Uncomplicated corrective surgery was undertaken.
\end{abstract}

Cor triatriatum sinister is a rare cardiac anomaly in which the left atrium is divided into upper and lower chambers by a transverse membrane. Communication between the upper and lower chambers is via a stenotic fenestration in the membrane which tends to lead to a rise in pulmonary venous pressure. The upper chamber usually communicates with the right atrium via a patent foramen ovale or atrial septal defect so that the clinical features may simulate anomalous pulmonary venous connection. Less frequently the foramen ovale communicates with the distal chamber so that the clinical features mimic mitral stenosis.

The diagnosis of rheumatic mitral stenosis is now rare in the white population with the great decline in the incidence of rheumatic fever. We report the case of a young man presenting ten years after an episode of typical Sydenham's chorea with atrial fibrillation and left atrial enlargement that initially was thought to be due to rheumatic mitral stenosis.

Case report

A 22 year old white man who participated in the strenuous pastime of mediaeval jousting presented with exertional breathlessness. Ten years earlier he had consulted a neurologist with choreiform movement associated with facial grimacing so typical of Sydenham's chorea that he agreed to be admitted for the benefit of the medical students and his case was later presented locally (West Country Physicians Meeting, 1983).

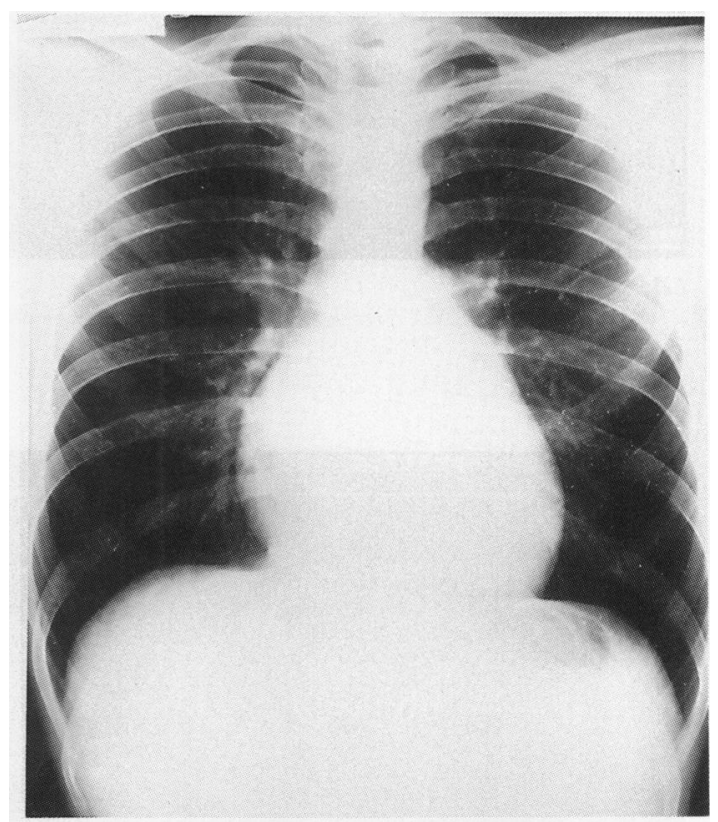

Figure 1 Chest radiograph in posteroanterior projection showing left atrial enlargement.

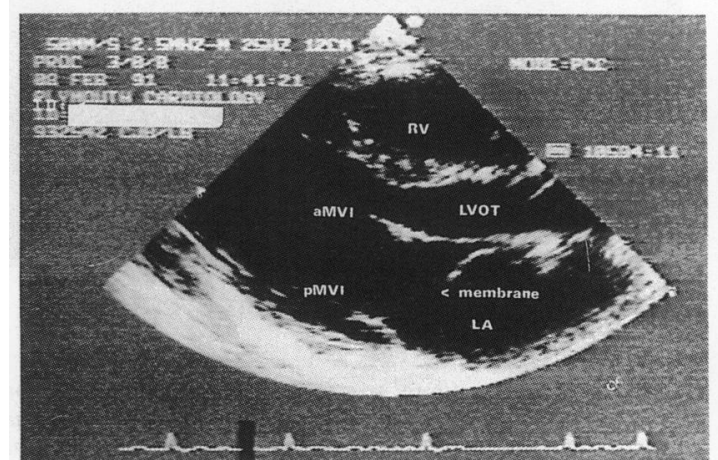

Figure 2 Cross sectional transthoracic echocardiogram (parasternal long axis view), showing an enlarged left atrium subdivided by a fibromuscular membrane (arrowed). LA, left atrium; LVOT, left ventricular outflow tract, aMVl, anterior mitral valve leaflet; $p M V l$, posterior mitral valve leaflet; $R V$, right ventricle.

\author{
Correspondence to \\ Dr C J Burrell, Departmen \\ of Cardiology, Derriford \\ Hospital, Plymouth PL6 \\ $8 \mathrm{DH}$. \\ St Geor \\ The Royal London \\ Derriford Hospital, \\ Plymouth
}


Preliminary investigation showed him to be in atrial fibrillation with radiographic evidence of left atrial enlargement (fig 1), though it was noted that the left atrial appendage seemed normal. At auscultation a loud first heart sound and a variable diastolic murmur was heard, leading to the provisional diagnosis of rheumatic mitral stenosis. However, transthoracic echocardiography showed a normal mitral valve with a fibrous membrane dividing the left atrium into upper and lower chambers (cor triatriatum sinister) (fig 2).

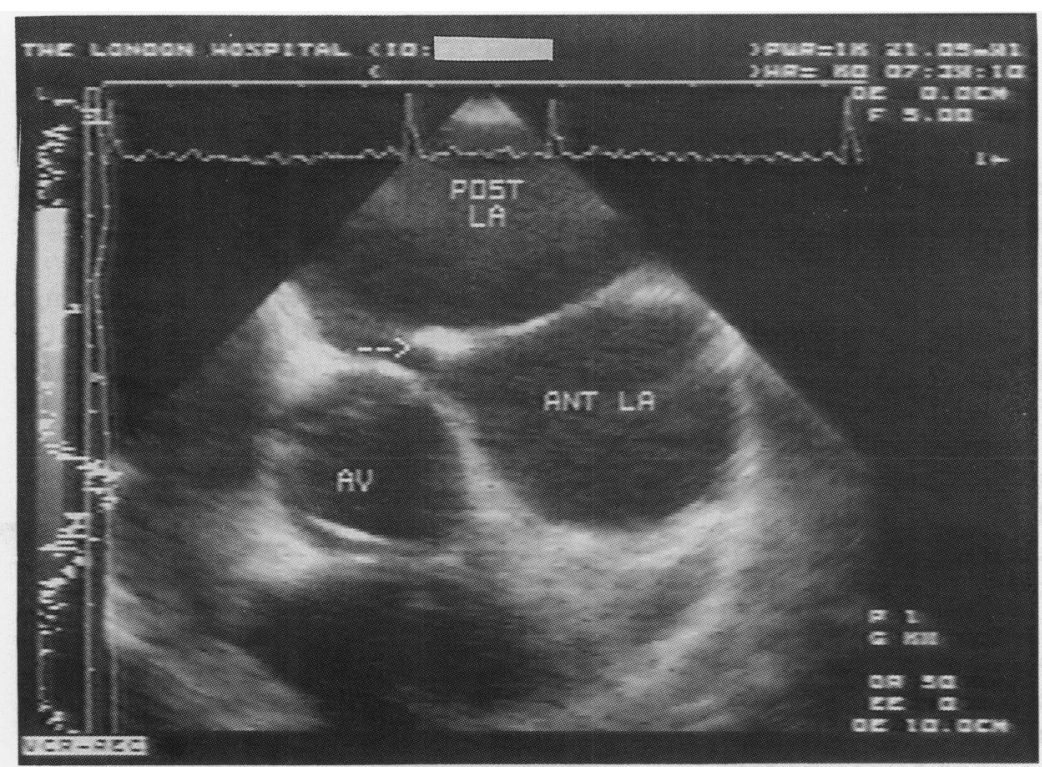

(A)

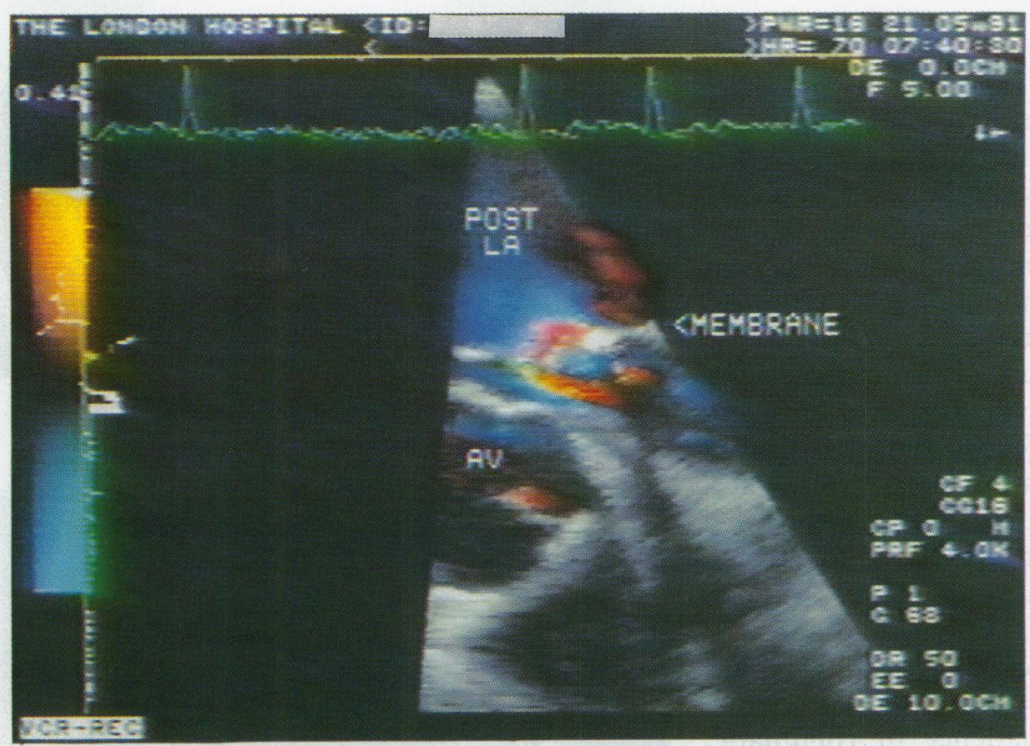

(B)

Figure 3 Cross sectional transoesophageal echocardiograms. (A) Horizontal section at the level of the aortic valve $(A V)$ just below the level of the left atrial appendage. $A$ defect (arrowed) is seen towards the medial side of the membrane, adjacent to the aortic root. Just below this level the membrane continued posteriorly and was attached to the posterior aspect of the atrial septum. AV, aortic valve; POST LA, posterior chamber of left atrium; ANT LA, anterior chamber of left atrium. (B) Colour flow map at the same level as $3 A$. There was a zone of flow acceleration in the posterior chamber, shown by serial colour aliasing, leading to a jet that passed through the defect in the membrane (arrowed) towards the mitral valve. AV, aortic valve; POST LA, posterior chamber of left atrium.
Cross sectional transoesophageal echocardiography showed the anatomy in detail (fig 3A). There was a small defect with a calcified rim on the medial side of the membrane adjacent to the wall of the aortic root. The pulmonary veins all drained into the upper chamber and colour flow doppler showed a high velocity jet from the upper chamber through the defect towards the mitral valve (fig 3B). The diagnosis and absence of associated cardiac abnormalities were confirmed by biplane cardiac angiography of the right and left heart (with a late phase pulmonary angiogram). Saturations were normal and pressures were recorded as follows $(\mathrm{mm} \mathrm{Hg})$ : pulmonary artery $35 / 14$, pulmonary capillary wedge 12 (mean), left ventricle 140/0-2.

At operation via a median sternotomy, hypothermic cardiopulmonary bypass was established and an incision was made in the left atrium at the Sondergaard sulcus, just posterior to the interatrial groove. The findings exactly matched the echocardiographic appearances. There was a thin fibromuscular septum subdividing the left atrium. It had an oval orifice ( $1 \mathrm{~cm}$ diameter) with calcified margins between the large upper and smaller lower chambers. The pulmonary veins drained separately into the upper chamber with which the foramen ovale also communicated. There was no atrial septal defect and no thrombus was present. The membrane was excised completely via the fenestration and though the mitral orifice was quite large, the valve apparatus was normal and shown to be competent. The left atrium was closed and the heart was defibrillated into sinus rhythm. The postoperative course was uncomplicated.

Three months later he was symptom free, and in sinus rhythm; he had not returned to mediaeval jousting. He had had a recurrence of chorea and his previous medication had been restarted.

\section{Discussion}

Cor triatriatum sinister accounts for only $0 \cdot 1 \%$ of all congenital cardiac anomalies. The anomaly, first described in $1868,{ }^{1}$ about two hundred years after Thomas Sydenham's description of childhood chorea, consists of an abnormal fibromuscular membrane dividing the left atrium into upper and lower chambers. There are two main variants depending on the position of the foramen ovale in relation to the membrane. When the upper chamber communicates via a patent foramen ovale (or an atrial septal defect) with the right atrium, obstructed total anomalous pulmonary venous drainage may be mistakenly diagnosed. When there is communication between the lower chamber and the right atrium cor triatriatum can mimic mitral stenosis.

In normal embryological development the common pulmonary vein originates between the fifth and eighth weeks of life as a midline structure below the septum primum. The normal distal connection of the pulmonary veins results from the incorporation of this structure into the left atrium. The embryo- 
logical basis for the anomaly remains uncertain but it may be due to a failure of incorporation, an overgrowth of the septum primum, entrapment of the common pulmonary vein, or indeed a combination of these..$^{2-4}$

Diagnosis, with evidence of increased pulmonary venous pressure, is most commonly made in the first year of life; our patient did not present until he was 22 , perhaps because the fenestration in the anomalous membrane was larger than usual $(1 \mathrm{~cm}$ diameter). Fenestrations can be as small as 1 to $2 \mathrm{~mm}$ in diameter. Associated congenital cardiac abnormalities are common, particularly atrial septal defect and true anomalous pulmonary venous drainage; ${ }^{56}$ a persistent left superior vena cava has also been observed quite frequently. ${ }^{67}$

In the past, accurate preoperative elucidation of the anatomy has depended upon left and right heart catheterisation with pulmonary angiography to show the membrane during the late phase in the right anterior oblique projection. Recent advances in echocardiographic techniques and particularly the application of transoesophageal echocardiography, however, allowed us to make complete preoperative assessment non-invasively; cardiac catheterisation did not contribute additional information.

Surgical treatment consists of excision of the fibromuscular membrane. The surgical approach is made directly into the upper chamber of the left atrium or, in smaller patients, via the right atrium, particularly if there is an associated atrial septal defect. However, this approach can make closure of the patent foramen ovale or repair of the atrial septal defect difficult and the mitral valve apparatus may be damaged. In infancy, surgical correction of isolated cor triatriatum can be associated with a high mortality (up to $40 \%$ ) but long term results after successful correction are usually excellent. ${ }^{56}$

Rheumatic fever is now rare, so the diagnosis of Sydenham's chorea in this patient was in itself of interest. The association between this condition and a congenital abnormality that may mimic mitral stenosis was presumably a chance one. We are not aware of any association between cor triatriatum sinister and choreiform movement.

We thank Mr C T Lewis for his help and expertise in the management of this patient and Mr D J Morris and Mrs Roach (Derriford Hospital, Plymouth) for their assistance.

1 Church WS. Congenital malformations of the heart. Abnormal septum in the left auricle. Trans Path Soc London 1868;19:188-99.

2 Van Praagh R, Corsini I: Cor triatriatum. Pathologic anatomy and a consideration of morphogenesis based on 13 post mortem cases and a study of normal development of the pulmonary vein and atrial septum in 83 puman embryos. Am Heart J 1969;78:379-405.

3 Marin-Garcia J, Tandon R, Lucas RV Jr, Edwards JE. Cor triatriatum. Study of 20 cases. Am J Cardiol 1975;35: 59-66.

4 Thilenius OG, Bharati S, Lev M. Subdivided left atrium. An expanded concept of cor triatriatum sinistrum. $A m$ J An expanded concept of

5 Richardson JV, Doty DB, Siewers RD, Zuberbuhler JR. Cor triatriatum (subdivided left atrium). J Thorac CarCor triatriatum (subdivided
diovasc Surg 1981;81:232-8.

6 Rodefeld MD, Brown JW, Heimansohn DA, et al. Cor triatriatum: clinical presentation and surgical results in 12 patients. Ann Thorac Surg 1990;50:562-8.

Gharagozloo F, Bulkley BH, Hutchins GM. A proposed pathogenesis of cor triatriatum. Impingement of the left superior vena cava on the developing left atrium. Am 